\title{
«OPTICAL COHERENCE TOMOGRAPHY» EN DESGARRO DEL EPITELIO PIGMENTARIO RETINIANO
}

\section{«OPTICAL COHERENCE TOMOGRAPHY» OF RETINAL PIGMENT EPITHELIAL TEARS}

\author{
TORRÓN-FERNÁNDEZ-BLANCO C ${ }^{1}$, FERRER-NOVELLA E ${ }^{1}$, RUIZ-MORENO O ${ }^{1}$, \\ HONRUBIA-LÓPEZ FM ${ }^{1}$
}

\section{RESUMEN}

Casos clínicos: Se presentan 3 pacientes con desgarro de epitelio pigmentario retiniano (EPR). En dos de ellos, éste se produjo de manera espontánea, estando el tercero en relación con la fotocoagulación de una neovascularización extrafoveal. Se realizaron angiografías (AGF) y estudio con tomografia óptica de coherencia (OCT) que confirmaron el diagnóstico.

Discusión: Existen pocos estudios sobre OCT en desgarro del EPR. Las principales características son: interrupción de la línea hiperreflectante del EPR, hiperreflectividad de la coroides en la zona de ausencia de EPR y doble capa de EPR donde éste está replegado. Es frecuente el desprendimiento de EPR y neurosensorial asociado.

Palabras clave: Desgarro EPR, OCT, desprendimiento de EPR, DMAE.

\begin{abstract}
Case reports: Three cases of pigmentary epithelial tears are described in this study. One of them was related to previous extrafoveal photocoagulation, whereas the other two were idiopathic. Fluorescein angiography (FA) and optical coherence tomography (OCT) were performed to confirm the clinical diagnosis.

Discussion: Few studies using OCT to assess pigmentary retinal epithelial (RPE) tears have been published in the world literature. The main findings of our study included hyper-reflectant line breaks, choroidal hyper-reflectance due to the lack of RPE and RPE double layering where it was folded. Images were frequently associated with RPE tears and neurosensorial retinal detachment (Arch Soc Esp Oftalmol 2007; 82: 245-250).
\end{abstract}

Key words: RPE tear, OCT, RPE detachment, ageassociated macular degeneration.

Recibido: 15/9/06. Aceptado: 23/3/07.

Servicio de Oftalmología. Hospital Univrsitario Miguel Servet. Zaragoza. España.

1 Doctor en Medicina.

Correspondencia:

C. Torrón

Vía Hispanidad, $35,1 .^{\circ}, 1{ }^{\circ} \mathrm{A}$

50012 Zaragoza

España

E-mail: clemenciatorron@terra.es 


\section{INTRODUCCIÓN}

El desprendimiento seroso del epitelio pigmentario retiniano (EPR) es habitual en la degeneración macular asociada a la edad (DMAE)exudativa; una complicación no infrecuente es el desgarro del EPR, que provoca una disminución brusca de visión de mal pronóstico $(1,2)$.

Presentamos los resultados del estudio con OCT y AGF en tres pacientes con desgarro de EPR y DMAE exudativa.

\section{CASOS CLÍNICOS}

\section{Caso 1}

Mujer de 79 años pseudofáquica. Acudió a urgencias por disminución de agudeza visual (AV) en ojo derecho (OD) de 2 meses de evolución. La AV era de 0.2 y en el fondo de ojo existía una zona central de atrofia de EPR , y una zona opaca adyacente de levanta- miento temporal superior, compatible con desgarro de EPR. La AGF demostró la hiperfluorescencia de transmisión central con punto caliente nasal a la misma y otra de hipofluorescencia temporal superior (figs. 1A y B). El examen con OCT mostró la hiperreflectividad de la coroides por la ausencia de EPR en la zona nasal, doble banda de EPR, zonas de hiporreflectividad ocasionadas por edema intra y sub-retiniano (figs. $1 \mathrm{C} \mathrm{y}$ D). Se realizaron dos sesiones de Terapia fotodinámica con verteporina, con buena respuesta inicial (AV 0,3), pero 3 meses después del $2 .^{\circ}$ tratamiento, la AV había descendido nuevamente hasta contar dedos observándose en fondo de ojo una cicatriz macular disciforme.

\section{Caso 2}

Varón de 82 años sin antecedentes de interés que acudió a urgencias por disminución de AV en OD de tres días de evolución. A la exploración la AV era de 0,2 , en fondo de ojo existía una amplia zona de atrofia temporal y una zona oscura de desprendi-
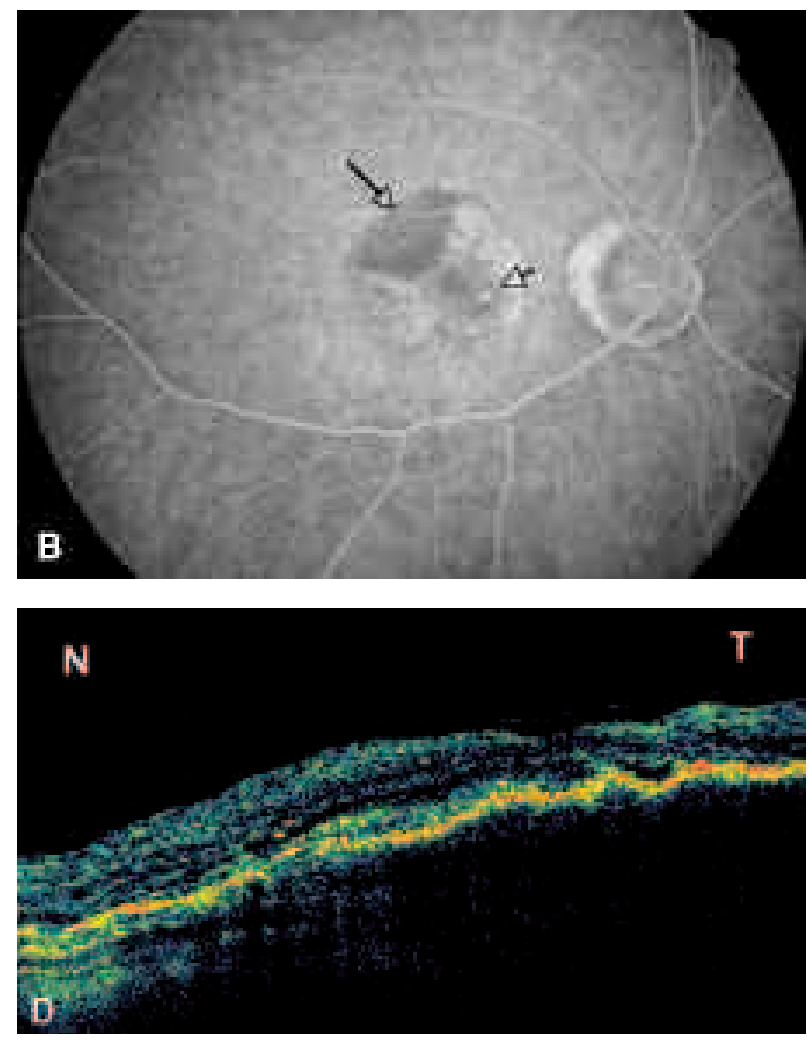
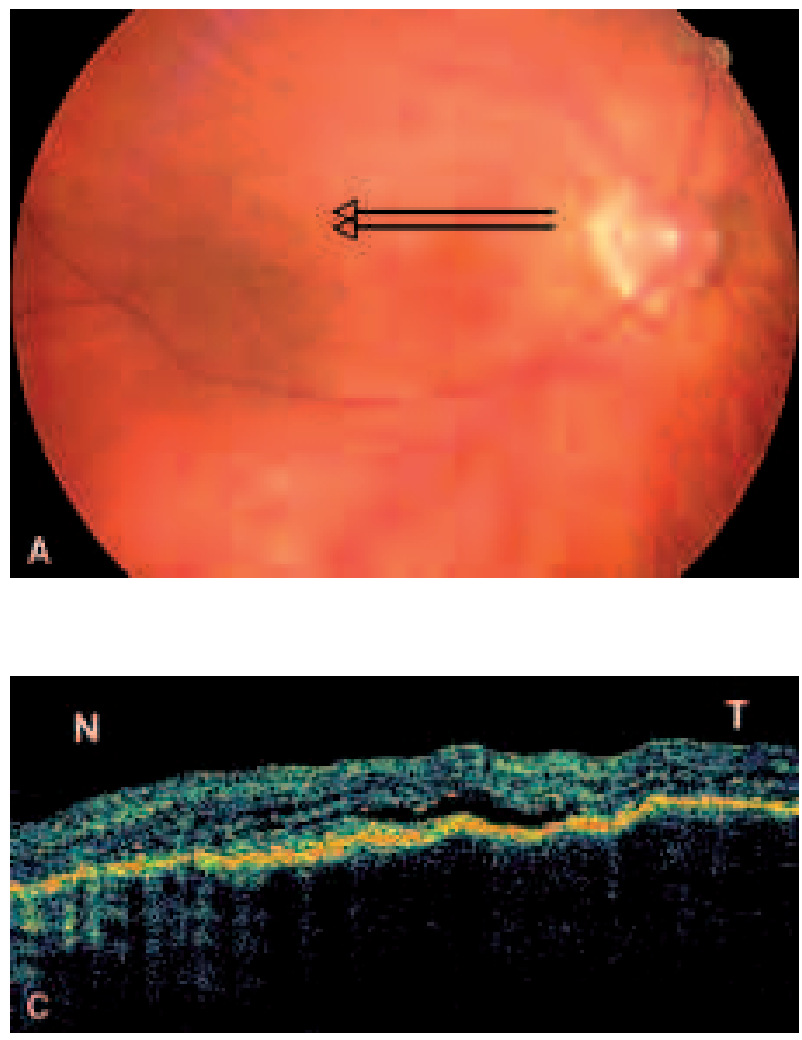

Fig. 1: Caso número 1. A. Retinografia: zona central de atrofia y levantamiento temporal superior. B. Angiografia fluoresceínica: hiperfluorescencia de transmisión central con punto caliente nasal (flecha pequeña), hipofluorescencia temporal superior (flecha grande). C y D tomografía óptica de coherencia de nasal (N) a temporal (T). 
miento de EPR, compatible con desgarro de EPR. La AGF confirmó el diagnóstico, mostrando hiperfluorescencia precoz en la zona temporal atrófica y en el centro de la zona hipofluorescente central, en fases tardías existía aumento de la intensidad y difusión (figs. 2 A-C). La OCT mostraba claramente el desgarro de EPR. En la zona temporal hiperreflectividad de la coroides por la ausencia de EPR, hiporreflectividad sobre ella por el desprendimiento neurosensorial. En la zona nasal doble banda de EPR y elevación de EPR con hiporreflectividad subyacente por el desprendimiento de EPR (figs. 2 D y 3). El paciente declinó cualquier tratamiento. Dos meses después la AV había descendido a movimientos de mano, cuatro meses después era de percepción y proyección de luz, obsevándose en fondo de ojo una hemorragia vítrea organizada.
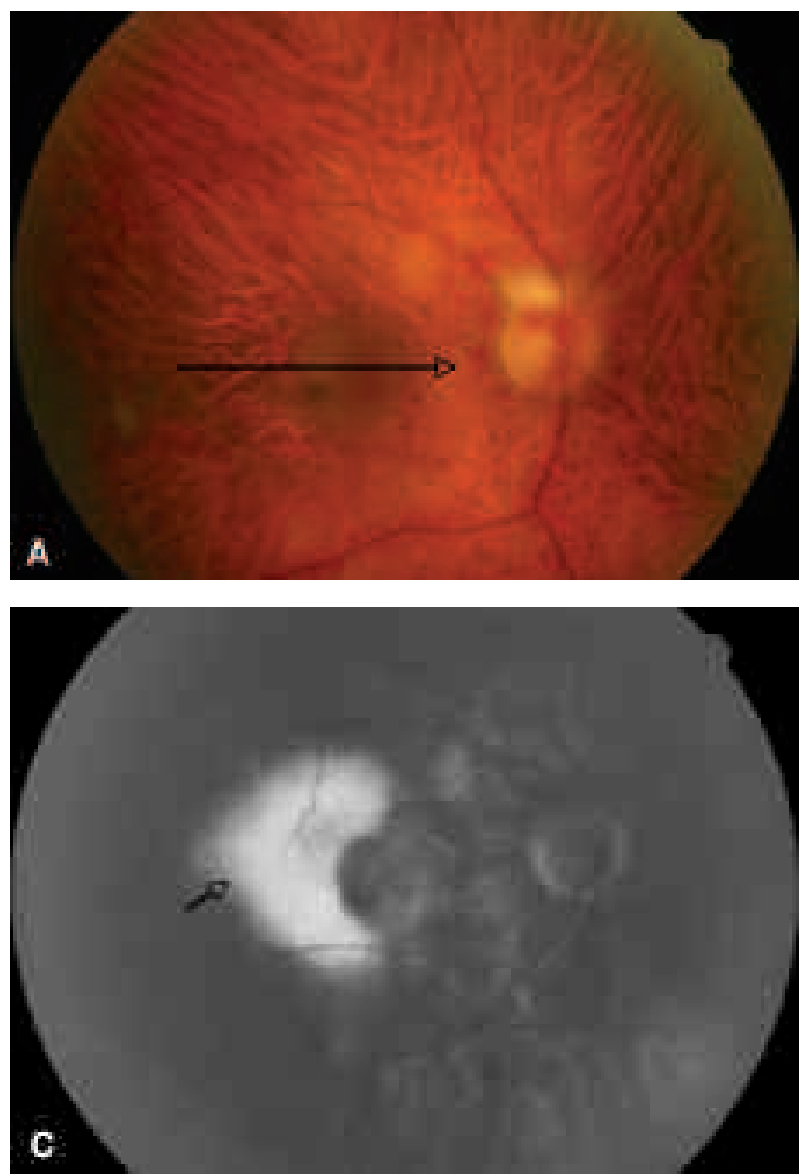

\section{Caso 3}

Mujer de 78 años, con antecedentes de fotocoagulación láser por edema macular diabético y drusas blandas en ambos ojos. Acudió a urgencias por disminución visual, la AGF demostró una membrana neovascular extrafoveal de pequeño tamaño que fue tratada con fotocoagulación láser con buen resultado inicial (AV mejoró de $0,1$ a 0,3$)$.Seis meses después, la AV había descendido a 0,05, observándose en fondo de ojo una lesión atrófica nasal junto a zona oscura de levantamiento y pliegues, sugestiva de desgarro del EPR. La AGF y la OCT confirmaron el diagnóstico (fig. 4). No se realizó ningún tratamiento, siendo la $\mathrm{AV}$ en la última exploración 6 meses después de 0,02 .
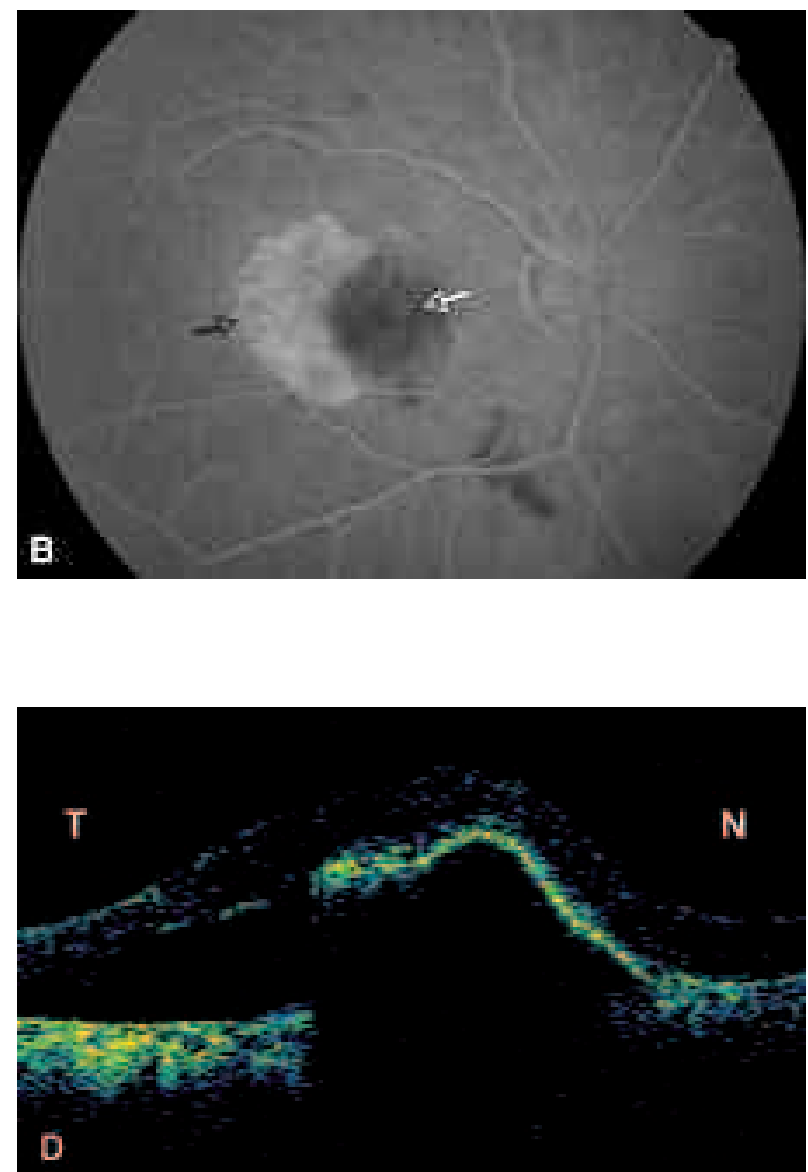

Fig. 2: Caso número 2. A. Retinografia: atrofia temporal, levantamiento central. B y C. Angiografia fluoresceínica: hiperfluorescencia con difusión tardía (flecha negra), hipofluorescencia central (flecha blanca). D. Tomografía óptica de coherencia de temporal (T) a nasal (N). 


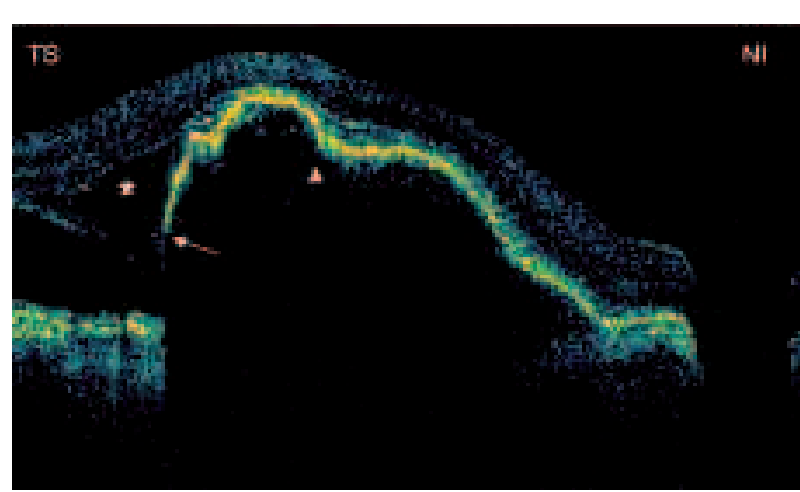

Fig. 3: Caso número 2. Tomografía óptica de coherencia: a la izquierda de la imagen hiperrreflectividad profunda en la zona de ausencia de epitelio pigmentario retiniano (EPR), interrupción del EPR (flecha grande), desprendimiento irregular del EPR (flecha pequeña), desprendimiento neurosensorial (asterisco).
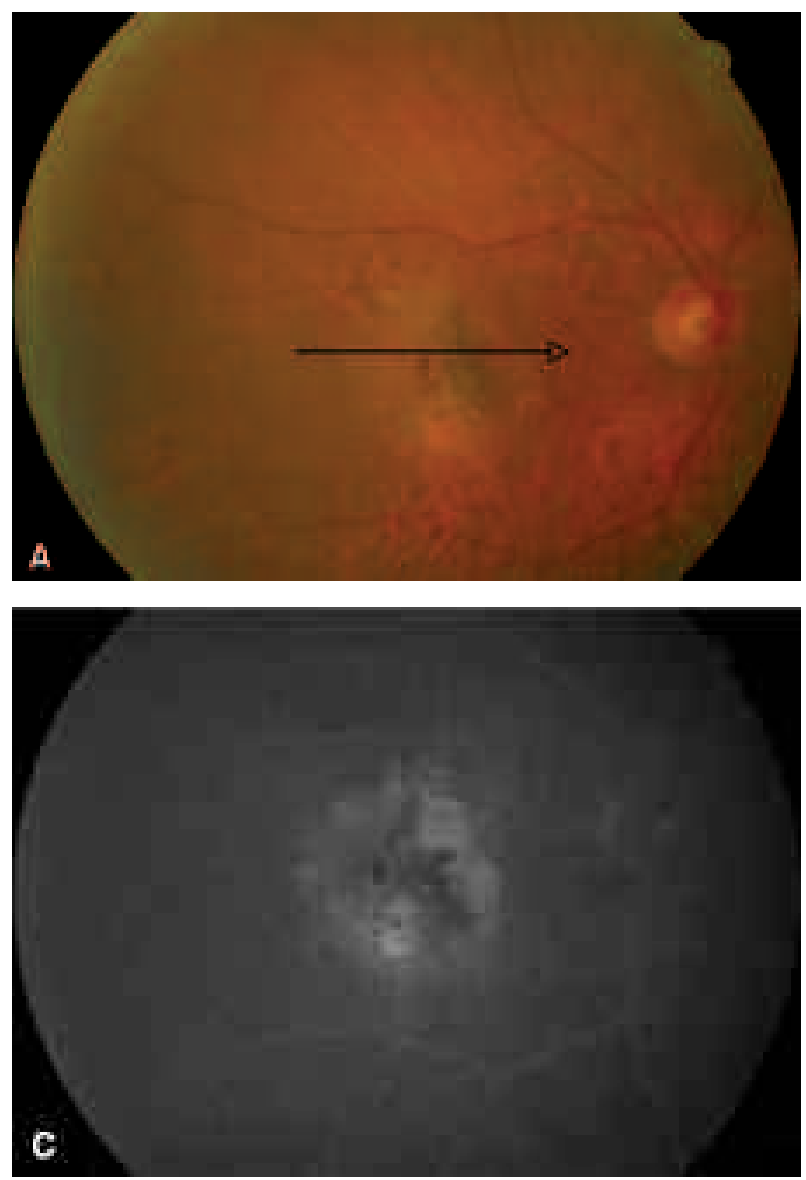

\section{DISCUSIÓN}

El desgarro el EPR es una grave complicación de la degeneración macular asociada a la edad (DMAE), que puede ocurrir ya sea espontáneamente o después de diversos tratamientos para la neovascularización frecuentemente asociada: fotocoagulación láser, terapia fotodinámica, pegaptanib intravítreo (1-3). Debe diferenciarse de la DMAE atrófica o neovascularización subretiniana (1). La AGF y en los últimos años la OCT, son de gran utilidad para realizar el diagnóstico (1-5). Angiográficamente es típica la hiperfluorescencia de transmisión producida por la coroides denudada y el bloqueo de fluorescencia inducido por el EPR retraído. Las dos zonas están separadas por una línea bien definida que es el borde libre del EPR replegado. Con frecuencia
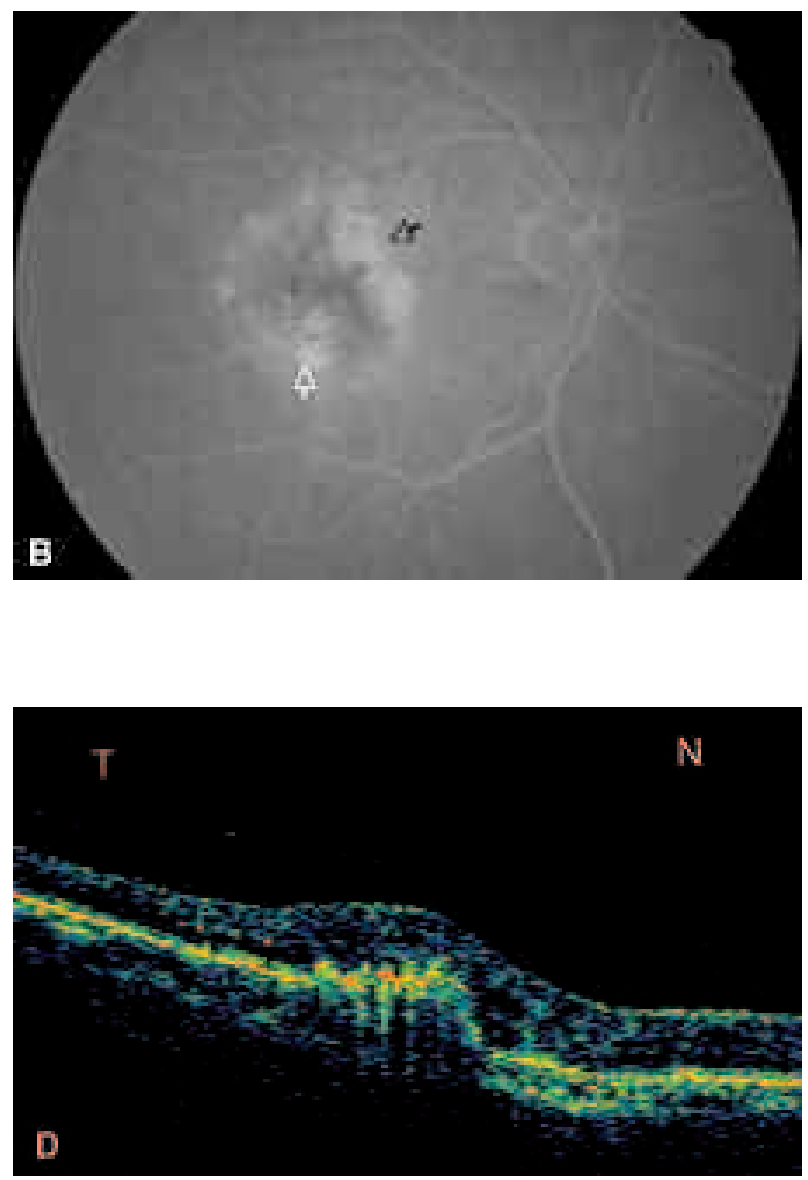

Fig. 4: Caso número 3. A. Retinografia: microhemorragias, atrofia nasal, levantamiento central con pliegues, cicatrices láser. B y C. Angiografia fluoresceínica: hiperfluorescencia nasal (flecha negra), cicatriz láser (flecha blanca) hipofluorescencia central. D. Tomografía óptica de coherencia de temporal $(T)$ a nasal $(N)$ : hiperreflectividad central, interrupción del epitelio pigmentario, hiperreflectividad profunda nasal. 
existe además un desprendimiento de EPR o neurosensorial (figs. 1-3).

Existen escasas publicaciones sobre OCT en desgarro de EPR. Giovannini (5) publicó en el 2000 la serie más numerosa de 16 pacientes estudiados mediante esta técnica. La OCT consiguió identificar un desprendimiento de epitelio pigmentario de aspecto peculiar, no cupuliforme, con interrupción de la banda del EPR en todos los casos. La coroides denudada presentaba una hiperreflectividad profunda por la mayor penetración del haz en ausencia de la pantalla del EPR. El EPR retraído presentó intensa hiperrreflectividad por la duplicación de la capa. En nuestros tres pacientes pueden apreciarse estos hallazgos. En el caso número 2 con muy pocos días de evolución, la OCT permite apreciar el desprendimiento irregular del EPR, además de un desprendimiento neurosensorial en relación con una membrana neovascular oculta (fig. 3). Los otros dos pacientes llevaban varios meses de evolución por lo que estos hallazgos son menos llamativos aunque también apreciables (figs. 1 y 4). Giovannini (5) observó como única diferencia entre el estadío agu- do y cicatricial, la ausencia en éste de desprendimiento neurosensorial.

Consideramos que la OCT es una técnica muy útil para el diagnóstico y seguimiento de pacientes con desgarro de EPR y DMAE exudativa.

\section{BIBLIOGRAFÍA}

1. Krishan NR, Chandra SR, Stevens TS. Diagnosis and pathogenesis of pigment epithelial tears. Am J Ophthalmol 1985;100: 698-707.

2. Yeo JH, Marcus S, Murphy RP. Retinal pigment epithelial tears. Patterns and prognosis. Ophthalmology 1988; 95: 8-13.

3. Michels S, Aue A, Simader C, Geitzenauer W, Sacu S, Schmidt-Erfurth U. Retinal pigment epithelium tears following verteporfin therapy combined with intravitreal triamcinolone. Am J Ophthalmol 2006; 141: 396-398.

4. Dhalla MS, Blinder KJ, Tewari A, Hariprasad SM, Apte RS. Retinal pigment epithelial tear following intravitreal pegaptanib sodium. Am J Ophthalmol 2006; 141: 752754.

5. Giovannini A, Amato G, Mariotti C, Scassellati-Sforzolini. Optical coherence tomography in the assessment of retinal pigment epithelial tear. Retina 2000; 20: 37-40. 